the combinations of polymorphisms that exist in the population.

The concept of robustness suggests an answer. Biochemical pathways are constructed in such a way that their output is relatively insensitive to the types of variations that occur in natural polymorphisms. One might say that this result is inevitable, because any polymorphisms that frequently resulted in combinations that were lethal or caused infertility would be eliminated. However, Barkai and Leibler did not limit themselves to naturally occurring polymorphisms. If their simulations apply to the real world, their results imply that at least this particular pathway is insensitive to almost all polymorphisms.

How can we begin to think about the implications of genetic diversity on the biology of outbred organisms? Simple concepts that come to mind are those of complex traits and genetic modifiers, ideas that ultimately centre on one or a few genes. Rather than focusing attention on individual proteins, Barkai and Leibler suggest focusing attention on the collection of proteins that constitute a biochemical pathway. To generalize their concept, one could imagine that all pathways are constructed to be insensitive to fine-tuning. Although this hypothesis is certainly not justified by consideration of only one pathway, and that by simulation only, the idea leads to many interesting questions that are ultimately subject to experiment.

Obviously, the first question is what the consequences are if diversity is generated in a real cell. A second is the issue of what constitutes a pathway. The chemotaxis pathway considered by Barkai and Leibler is composed of 12 proteins sitting in a cytoplasmic sea of several thousand other proteins. Is the set that we consider a pathway the same as the set that evolution has considered a pathway? How could we tell? A result of Barkai and Leibler's suggests a test. They found that the ability to adapt to basal response was robust but that the time required to adapt was not. The set of proteins that constitute one pathway and affect one behaviour might be tested to see if they are part of or distinct from the set that constitute another pathway (and affect a second behaviour), in the following way. Introduce variation in one set of proteins that perturbs a non-robust parameter of behaviour 1; then determine whether this change affects a non-robust parameter of behaviour 2 .

A further question is what the robust parameters of other pathways might be. An answer would identify the most important output of a pathway, the characteristic that was selected by evolution to be robust. Finally, do apparently redundant pathways exist because one has been selected to be robust for one output whereas another has been selected to be robust for a different output, thereby allowing the cell to be robust to both outputs? It might be fruitful to consider genetic disease as due to those rare alleles that map outside the zone of robust variation.

Readers should turn to the paper itself on page 913 to find out in more detail about the authors' ideas. I am sure they will think of many more questions that can now be formulated as a result of this powerful concept of robustness.

Leland Hartwell is in the Fred Hutchinson Cancer Research Center, 1124 Columbia Street C2-023,

Seattle, Washington 98104, USA.

\title{
Biological oceanography
}

\section{Sulphur, climate and the microbial maze}

\section{Gillian Malin}

t might seem strange that marine algae and climate are linked, but there is a connection and it occurs through the production of a compound which breaks down to release volatile dimethyl sulphide - DMS, $\left(\mathrm{CH}_{3}\right)_{2} \mathrm{~S}$ - and acrylic acid. Formation of the volatile trace gas has implications that go far beyond the level of an individual cell or microbial population, because DMS is a key compound in the global sulphur cycle and its oxidation products influence atmospheric acidity, as well as cloud formation and the Earth's temperature ${ }^{1}$. The compound in question is dimethylsulphoniopropionate (DMSP), which algae synthesize to help maintain their osmotic balance with sea water.

Papers on pages 891 and 894 of this issue advance our understanding of how DMS is produced in the marine environment. Gage et $a l^{2}$ reveal the biochemical pathway for DMSP synthesis in a diverse group of marine algae. Wolfe and co-workers ${ }^{3}$ provide fresh insight into the involvement of grazing microzooplankton in releasing DMSP from marine phytoplankton cells.

The main sources of DMSP in the natural environment are marine phytoplankton blooms, where high levels of primary production and biomass occur on a timescale of weeks (Fig. 1), and phytoplankton assem-

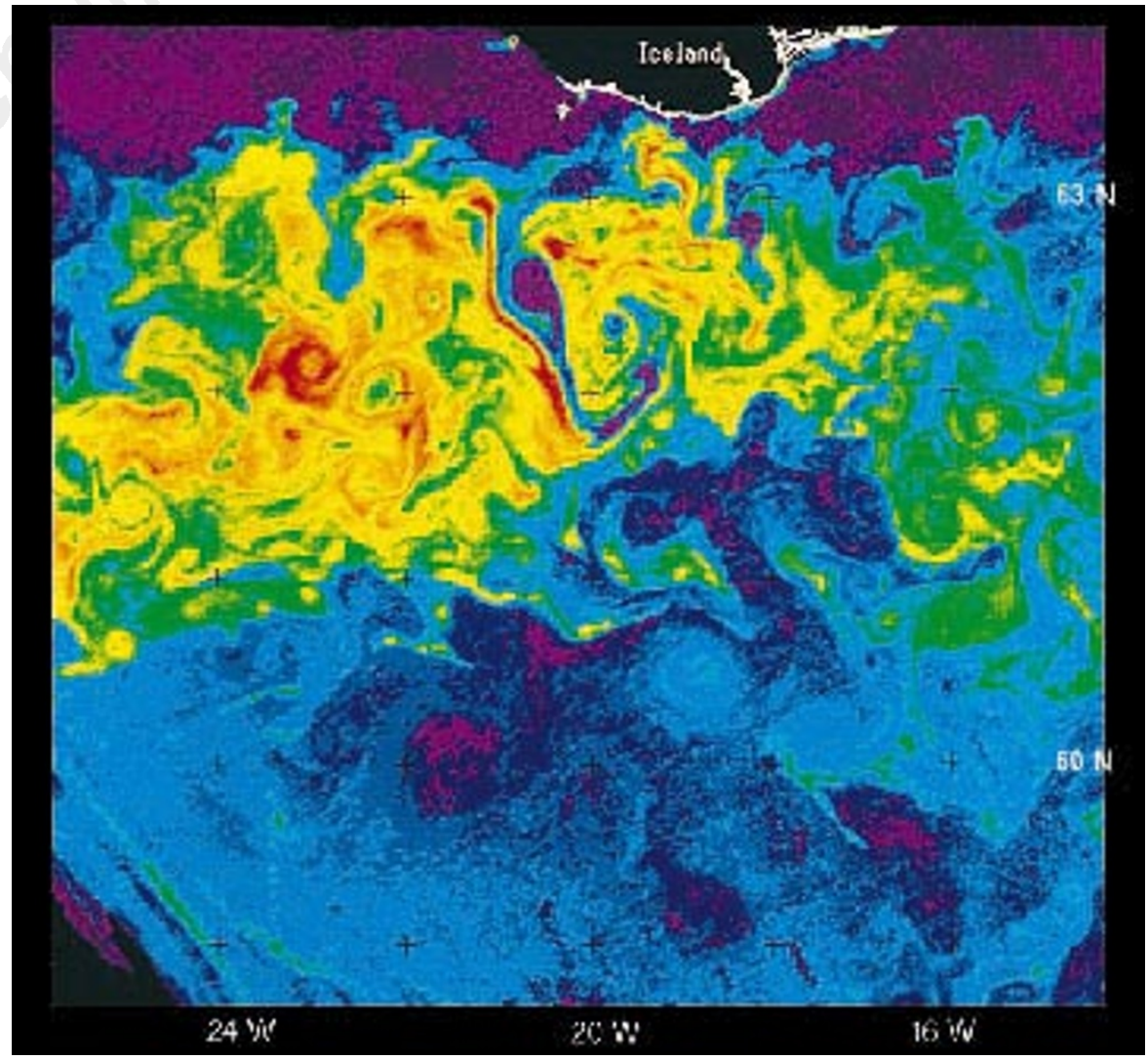

Figure 1 False-colour satellite image depicting the reflectance due to a large-scale bloom of Emiliania huxleyi in the northeast Atlantic, south of Iceland, in June 1991. Blooms such as this produce large amounts of dimethyl sulphide (DMS), the oxidation products of which influence atmospheric chemistry and climate. The rainbow colour scale represents high (red-orange) to low (blue-violet) levels of reflectivity of the upper layer of the sea. The reflectivity, which intensifies towards the end of a bloom, is due to the cells shedding coccoliths into the water column as they grow. (Image processed by S. Groom, Remote Sensing Data Analysis Service, Plymouth Marine Laboratory.) 


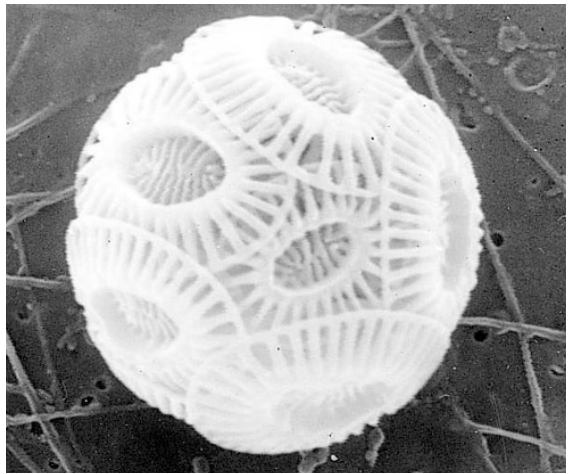

Figure 2 Scanning electron micrograph of a cell of the marine phytoplankton species Emiliania huxleyi, approximately $5 \mu \mathrm{m}$ in diameter. The outside of the cell is covered with ornate interlocking plates of calcium carbonate known as coccoliths. This organism contains high concentrations of dimethylsulphoniopropionate (DMSP), a precursor of DMS. The work discussed here ${ }^{2,3}$ shows the route by which DMSP is synthesized in marine algae, and how grazing results in its conversion to DMS and acrylic acid. (Micrograph courtesy of D. Harbour, SAHFOS, Plymouth.)

blages in the vast areas of the open oceans where steady-state populations exist for longer periods. Every millilitre of sea water contains a large and diverse microbial population of photosynthetic cells, zooplankton, bacteria and viruses. The processes by which DMSP is released from phytoplankton cells and DMS is produced are best depicted by a network of production, transformation and consumption which involves the entire marine microbial food web ${ }^{4}$. To date several key processes have been identified, although turnover rates and what controls them are not well understood. What is clear is that less than 10 per cent of the DMS in surface sea water ever enters the atmosphere. The rest is turned over by bacteria or oxidized by sunlight to form non-volatile products.

Grazing is a major route for the release of DMSP to sea water. In many regions unicellular microzooplankton are the principal herbivores, and they have a particularly important impact on small marine phytoplankton cells such as Emiliania huxleyi-a species that contains large amounts of DMSP (Fig. 2). These protozoa are highly selective in what they eat, and use chemical cues to select and reject prey ${ }^{5}$. Some DMSPcontaining microalgae have an enzyme known as DMSP lyase, which does not come into contact with intracellular DMSP during normal growth. Wolfe et al. ${ }^{3}$ propose that grazing mixes DMSP with the enzyme, thereby liberating DMS and acrylic acid, and then go further to suggest that DMSP may act as a defence precursor. Acrylic acid has signalling properties for a range of organisms, including bacteria, protozoa and fish, and the herbivorous microzooplankton used in the study actively retreated from gradients of the compound. In the sea this response might shift grazing pressure away from cells that contain high levels of DMSP. Wolfe and colleagues' report is the first of grazing-activated defence for a unicellular organism.

In their paper, Gage and co-workers ${ }^{2}$ describe a series of elegant biochemical experiments through which they have identified the intermediates in DMSP biosynthesis, including the novel compound dimethylsulphoniohydroxybutyrate (DMSHB). They show that the pathway in a common green seaweed is different from that found in higher plants, which suggests that these pathways evolved independently. However, macroalgae, which are typically found in the intertidal and subtidal zones of rocky shores, are thought to have only a small effect on the global sulphur cycle when compared with marine phytoplankton. With this in mind, Gage et al. went on to identify DMSHB in three phytoplankton species from different algal classes. DMSHB was readily catabolized when added to algal cultures, and the authors make the intriguing suggestion that it could be an additional precursor of DMS in vivo.

Marine organisms usually have a combination of organic osmolytes. DMSP is similar in both structure and function to the nitrogen-containing osmoregulatory compound glycine betaine (GBT). It has been proposed that, under conditions of nitrogen limitation $^{6}$, a common occurrence in the marine environment, organisms might preferentially change to making the sulphur-containing DMSP molecule. There is evidence from laboratory studies and a field study that such a
GBT-DMSP switch exists ${ }^{7}$, and Gage and colleagues now provide further support for the idea at the biochemical level. DMSP synthesis is initiated by a transamination reaction, and this pathway would be favoured in nitrogen-depleted cells, hence effectively favouring a switch to DMSP.

And what of the climate link? Global climate change is a big issue ${ }^{8}$, and there is general awareness of how human activities are changing the concentration and distribution of greenhouse gases. It is less well known that atmospheric aerosols, which are submicrometre particles or droplets, have a cooling effect on global temperature. The link with algae is that some of the marine DMS escapes into the atmosphere where it oxidizes rapidly to form aerosol particles; these absorb and reflect solar energy directly back into space, and scatter radiation indirectly by acting as cloud-condensation nuclei.

Natural aerosol levels have been substantially affected by anthropogenic sulphur, especially in the Northern Hemisphere. Nevertheless, DMS oxidation products remain the major source of aerosol particles over remote marine areas (that is, over most of the globe). Anything that disturbs the balance of the microbial ecology of DMS production, such as changes in wind speed that alter the upwelling of nutrients, or increases in sea-water temperature that change the spatial distribution of grazers relative to their phytoplankton prey, could affect how much of this sulphur compound is emitted to the air, and therefore affect climate.

In 1987 Charlson and colleagues ${ }^{1}$ sparked a flurry of research interest by proposing that

\section{Cell biology}

\section{Explorers deliver tea to the pole}

A few weeks ago, a report in these pages claimed that drinking green tea can prevent cancer (see Nature 387,$561 ; 1997$ ). Now, in the 13 June issue of Cell, Juan Mata and Paul Nurse report (Cell 89, 939-949; 1997) that another type of tea could help you to grow properly if you're a fission yeast, that is.

Immediately after cell division, the fission yeast Schizosaccharomyces pombe begins to grow. Initially, it extends only at the old tip that existed in the mother cell. But at a certain point in the cell cycle, it switches to bipolar growth, and its ends elongate directly away from one another. How does it do this?

To find out, Mata and Nurse studied yeast mutants in which the tips were not precisely opposed. Some of these mutants were bent, others were T-shaped, but all had defects in one of two tea (tip elongation aberrant) genes. Having cloned teal, the authors found that the teal

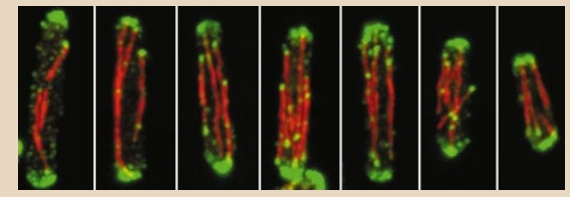

protein is tightly localized to both ends of a cell. Not only that but, using immunofluorescence staining, they saw that teal (green) colocalizes with the ends of tubulin (red) microtubules.

Mata and Nurse believe this to mean that teal acts as an end marker, directing the yeast's growth machinery to the cell poles. Teal is delivered to the poles by the microtubular cytoskeleton, the organization of which may be affected as a result. This produces a dynamic system in which the space of the cell is explored, then the two growing tips are identified and maintained by tea 1 - a kind of tea for two for yeast.

Alison Mitchell 
the connection between algae and clouds represents not only a climate linkage, but also a climate-regulating mechanism. Since then, as recent papers attest ${ }^{9,10}$, there has been a great deal of progress, and evidence in support of the climatic significance of DMS continues to grow. For instance, clear relationships have been shown to exist between high levels of DMS in the atmosphere, increased particle concentrations and enhanced cloud albedo. In all, it is clear that the myriad interactions between the components of the microbial food web and their physical and chemical environment fundamentally influence how much DMS is available for exchange with the atmosphere. But direct proof of the phytoplanktonDMS-climate feedback idea, whereby climate would affect the quantity of DMS emitted to the air, remains elusive.

The papers by Gage et $a l^{2}$ and Wolfe et al. ${ }^{3}$ are examples of how, by increasing our understanding of the marine microbial maze, we can inch closer to the ultimate goal of being able to predict how changes in environmental parameters might impinge on natural inputs of sulphur to the atmosphere.

Gillian Malin is in the School of Environmental

Sciences, University of East Anglia, Norwich

NR4 7TJ, UK.

e-mail: g.malin@uea.ac.uk

1. Charlson, R. J., Lovelock, J. E., Andreae, M. O. \& Warren, S. G. Nature 326, 655-661 (1987).

Gage, D. A. et al. Nature 387, 891-894 (1997).

3. Wolfe, G. V., Steinke, M. \& Kirst, G. O. Nature 387, 894-897 (1997)

4. Malin, G., Liss, P. S. \& Turner, S. M. in The Haptophyte Algae

(eds Green, J. C. \& Leadbeater, B. S. C.) 303-320 (Clarendon, Oxford, 1994)

Verity, P. G. J. Protozool. 38, 69-76 (1991).

6. Andreae, M. O. in The Role of Air-Sea Exchange in Geochemical Cycling (ed. Buat-Menard, P.) 331-362 (Reidel, Dordrecht, 1986). Liss, P. S., Hatton, A. D., Malin, G., Nightingale, P. D. \& Turner, S. M. Phil. Trans. R. Soc. Lond. B 352, 159-169 (1997).

8. Houghton, J. T. et al. (eds) Climate Change 1995: The Science of Climate Change (Cambridge Univ. Press, 1996).

9. Kiene, R. P., Visscher, P. T., Keller, M. D. \& Kirst, G. O. (eds) Biological and Environmental Chemistry of DMSP and Related Sulfonium Compounds (Plenum, New York, 1996).

10. Cox, R. A. et al. (eds) Phil. Trans. R. Soc. Lond. B 352, 139-254 (1997).
E nigmatic bursts of $\gamma$-rays from cosmic sources are detected about once a day from every direction on the sky. During their brief duration, typically between $10^{-2}$ and $10^{3}$ seconds ${ }^{1}$, these bursts often have the brightest $\gamma$-ray intensities ever seen. Now, after almost three decades of observation and speculation, the distance scale to $\gamma$ ray burst (GRB) sources seems at last to have been settled. Evidence has been growing at a remarkable rate in the past few months the most striking pieces appearing on pages 876 and 878 of this issue $e^{2,3}$ - to support the proposal that most, and perhaps all, of the bursts come from explosions involving neutron stars or black holes near the edge of our observable Universe. If this is indeed the case, GRB sources are among the most powerful events in the Universe. This was the view of most of the participants in a GRB workshop last month*.

In 1991, the Burst and Transient Source Experiment (BATSE), on board the Compton Gamma-Ray Observatory, began to accumulate what has now grown to almost 2,000 burst observations. These show us to be at the centre of a nearly homogeneous distribution of bursting sources, and the paucity of faint bursts implies that we are seeing almost to the edge of the source population ${ }^{4}$. These two features are natural consequences of any uniform source distribution in the simplest cosmological models for our expanding Universe ${ }^{5}$. Other suggestions, for

${ }^{*}$ Workshop on the Latest Developments in Gamma-Ray Bursts, Elba, Italy, 26-27 May 1997. example that GRBs are from relatively small, recurrent explosions on the surface of neutron stars now in the halo of our own Galaxy ${ }^{6}$, needed some ad hoc tuning of parameters such as delays in the onset of explosions. This made such models less appealing, even though they more easily explained certain controversial features of some bursts (such as cyclotron resonance spectral features, periodicities of several seconds, and possible burst source repetitions).

A 'smoking gun' was needed, to identify some GRB source with a familiar type of object which could determine the distancescale. The problem was that $\gamma$-ray telescopes such as BATSE have an angular resolution of several degrees, and so cannot pin down a burst location finely enough for other kinds of telescopes to identify a unique counterpart at other wavelengths. The Interplanetary Network of satellites, started in the 1970s, could triangulate the angular position of a burst from arrival-time differences at different satellites, but gave only a handful of arc-minute-sized error boxes, generally months after a burst - far too long an interval to allow the identification of other transient emissions associated with it.

Since its launch in 1996, the ItalianDutch satellite BeppoSAX has improved on both position and time constraints enormously: it can locate X-ray emission from a GRB to a few arc minutes, and then tell optical and radio telescopes where to look within a few hours. So far, it has identified three fading X-ray sources as probable GRB afterglows. The 28 February 1997 burst $^{7}$ was

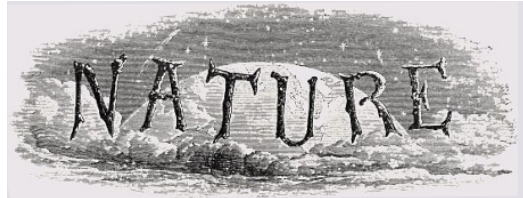

100 YEARS AGO

'Styles of the calendar' - At the approach of the end of a century, this subject naturally comes to the front again; but it has lately been somewhat unexpectedly raised to special prominence by the suggested probability of one at least of the Oriental countries of Europe adopting the usage which, on the initiative of Rome in 1582, all the western nations gradually accepted, England (we say advisedly England not Britain, because Scotland adopted it before the union even of the crowns) being the last in $\mathbf{1 7 5 2}$. America having been colonised by the western Europeans, and the United States having still been British colonies at the date last mentioned, the Gregorian style is universal in that continent. But eastern Europe, including Russia and all the nationalities of the Balkan peninsula, still adheres to the old Julian style; and this chiefly because the Christians of these countries belong to the Greek or Eastern Church, though it is difficult to see why this should restrain them from falling in with a change which has many conveniences, and would bring their dates into uniformity with those of the Latin, Teutonic, and Scandinavian nations - an object of increasing importance, as intercommunication is constantly becoming more frequent.

From Nature 24 June 1897.

\section{YEARS AGO}

Apart from its interest to Londoners and its inherent æsthetic merit, the statue of Eros, surmounting the Shaftesbury Memorial Fountain in Piccadilly Circus, London, has a scientific aspect. Originally unveiled in 1893, it is cast in aluminium alloy, the basal structure being of bronze. Its restoration, after a war-time exile, necessitated a thorough cleaning, the repair of a few defects, and the provision of a new bow and arrow. ... The cleaning was carried out with neutral soap and warm water, the surprising discovery being that there is no trace of corrosion arising from the past fifty years in the London atmosphere; with suitable care the original oxide film of the aluminium and the patina of the bronze have both been retained. ... The experience with the restoration of Eros should give an impetus to designers to make more use of these 'modern metals'.

From Nature 28 June 1947. 